\title{
Q Fever spondylodiscitis in the presence of endovascular infections: a case report
}

\author{
Austin SL Lim, MD, DPBO, Azizul AB Sali, MD, MMed, Jason PY Cheung *, MS, MD
}

Department of Orthopaedics and Traumatology, The University of Hong Kong, Hong Kong

Hong Kong Med J 2021;27:216-8

*Corresponding author: cheungjp@hku.hk

https://doi.org/10.12809/hkmj208622

\section{Case report}

In areas endemic for tuberculosis infections, the presence of infection on imaging and granulomatous inflammation on histology is often sufficient to start antituberculous pharmacotherapy. However, this may not be appropriate in cases of nontuberculous granulomatous infection. Rarer causes of spinal infection should be considered, especially if the clinical response is suboptimal. We present an unusual case of granulomatous spinal infection caused by Coxiella burnetii.

A 74-year-old man from southern China with no travel history presented with a 3-month history of low back pain in the absence of neurological deficit or constitutional symptoms. Co-morbidities included hypertension, diabetes mellitus, and hyperlipidaemia. There were no symptoms or signs of endocarditis. Initial plain radiographs showed lytic destruction of L4 and erosion of the L4-L5 disc space. Laboratory investigations revealed raised $\mathrm{C}$-reactive protein (CRP) of $27.6 \mathrm{~g} / \mathrm{dL}$ and erythrocyte sedimentation rate of $32 \mathrm{~mm} / \mathrm{h}$. Blood culture, Widal test for Salmonella, HIV testing and acid-fast bacilli growth in sputum and blood were all negative. There was no growth of fungus or brucellosis. Magnetic resonance imaging and computed tomography $(\mathrm{CT})$ revealed L4/5 spondylodiscitis with psoas and paravertebral abscesses (Fig 1), and a mycotic infrarenal abdominal aortic aneurysm measuring $7.3 \times 7.6 \times 5.7 \mathrm{~cm}$. He underwent endovascular stenting of the aneurysm. A CT-guided aspiration of the psoas abscess was negative on Ziehl-Neelsen and Grocott staining and tuberculous polymerase chain reaction. Histology confirmed granulomatous inflammation. After discussion with the microbiologist, the patient was prescribed intravenous ceftriaxone $2 \mathrm{~g}$ per day and metronidazole $1 \mathrm{~g}$ per day but switched to ertapenem $1 \mathrm{~g}$ per day due to a poor clinical response. These antibiotics were used as empirical therapy. Symptoms subsided and his CRP normalised to $<0.35 \mathrm{~g} / \mathrm{dL}$. He was discharged home and prescribed lifelong amoxicillin/clavulanic acid $375 \mathrm{mg} 3$ times daily, with levofloxacin $500 \mathrm{mg}$ once daily for the mycotic aneurysm.

At 6 months after discharge he presented with recurrent back pain along with radicular symptoms. Inflammatory markers were elevated with CRP $3.5 \mathrm{~g} / \mathrm{dL}$ and erythrocyte sedimentation rate $81 \mathrm{~mm} / \mathrm{h}$. The same cultures and serology were negative. Plain radiographs and $\mathrm{CT}$ showed increased bony destruction of L4. Magnetic resonance imaging revealed persistent retroperitoneal abscess with progressive spondylodiscitis (Fig 2). A CT-guided drainage of his right psoas abscess yielded negative culture results. Due to the neurological deterioration, an L3-L5 laminectomy with posterior instrumented spinal fusion was performed. Anterior column reconstruction was attempted by the vascular surgeon but scarring prevented safe access to the spinal column without aneurysmal injury. The L4-L5
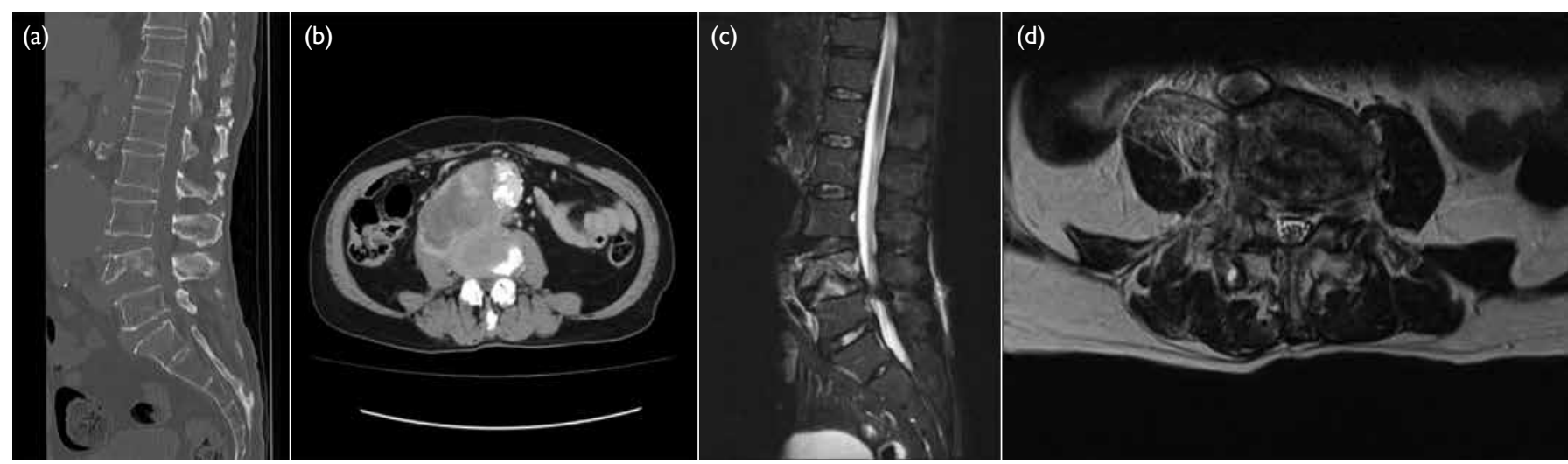

FIG I. A 74-year-old man with a 3-month history of low back pain. (a) Initial sagittal computed tomography scan showed L4 bony destruction. (b) Axial contrast computed tomography scan showed a mycotic aneurysm with extension to the L4/5 disc space and right psoas muscle. (c) Sagittal T2weighted magnetic resonance imaging showed L4/5 spondylodiscitis. (d) Axial contrast magnetic resonance imaging showed right psoas inflammatory changes 


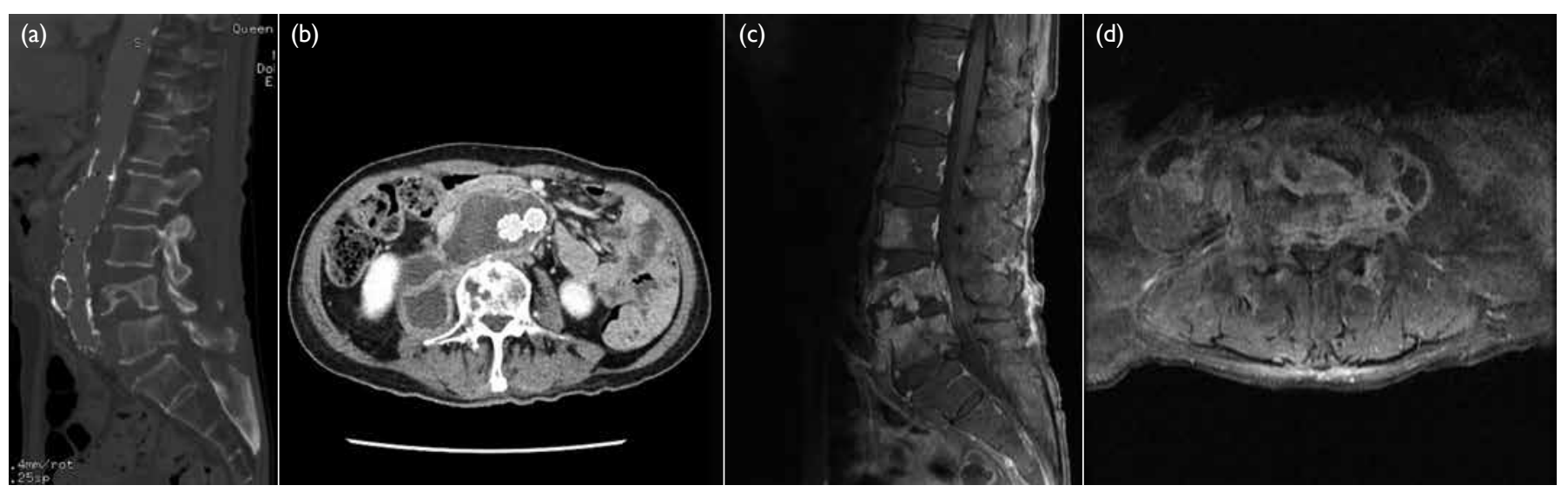

FIG 2. Same patient after failed pharmacotherapy for 6 months. (a) Sagittal computed tomography scan showed further L4 bony destruction. (b) Contrast axial computed tomography scan showed more extensive abscess formation around the aorta and the right psoas. (c) Sagittal contrast TI-weighted magnetic resonance imaging showed more extensive infection involving L3 as well. (d) Axial scan showed the infection spread to the left psoas and causing spinal stenosis

disc material revealed granulomatous inflammation and methicillin-resistant Staphylococcus aureus. He was treated as co-infection with tuberculosis and methicillin-resistant Staphylococcus aureus, and was given isoniazid $300 \mathrm{mg}$, rifampicin $450 \mathrm{mg}$, and ethambutol $800 \mathrm{mg}$ once daily, and linezolid $600 \mathrm{mg}$ every 12 hours. His inflammatory markers normalised but back pain persisted.

Serial radiographs showed implant failure with further destruction of L4 (Fig 3) due to the lack of anterior column support. Revision posterior instrumented fusion was performed 3 months after the initial surgery from T10 to the pelvis with cement augmentation after removal of the loosened L3-L5 implants. Intra-operative findings were friable tissue and osteoporotic bone. Histology still showed granulomatous inflammation but other cultures were negative. Due to the persistent infection with negative cultures, serology for C burnetii was performed and revealed increased phase I and phase II polyvalent antibodies with titre results of 3200 for both. The patient was diagnosed with chronic $\mathrm{Q}$ fever and was given lifelong hydroxychloroquine sulphate $200 \mathrm{mg}$ every 8 hours and doxycycline hyclate $100 \mathrm{mg}$ every 12 hours owing to the presence of the endovascular stent and spinal instrumentation. Serial monitoring of $\mathrm{Q}$ fever serology was performed every 6 months. At 2 years after surgery, he remains symptom free with no implant loosening.

\section{Discussion}

First described in 1937, Q fever is caused by $C$ burnetii and can be found worldwide with an overall prevalence of $10 \% .{ }^{1,2}$ Inoculation is through direct contact, ingestion or inhalation of contaminated materials. Traditionally it is thought that contact with cows, goats, sheep or cats causes this disease, but it is not always the case. In chronic $Q$ fever, endocarditis is the most common presentation, seen in $60 \%$ to $70 \%$ of cases. Other manifestations include hepatitis, pericarditis, myocarditis, vascular infections, and osteoarticular infection..$^{3}$ Diagnosis is usually by serological testing but can also be on smears or frozen tissue with Giemsa staining that reveals doughnut granulomas. Although serology shows only indirect evidence of infection, it is a simpler and reliable technique. In acute $\mathrm{Q}$ fever, testing is performed for antibodies against phase II antigens. In chronic $\mathrm{Q}$ fever, testing is for antibodies against phase I antigens. Diagnosis is confirmed if immunoglobulin G titre exceeds 1/800. Cultures are not commonly performed due to its high infectivity and is not available in our laboratory.

Osteoarticular Q fever infections are rare. One study from France showed that only $7.3 \%$ of all their patients with $\mathrm{Q}$ fever manifested with osteoarticular infection. ${ }^{4}$ The most common form of $\mathrm{Q}$ fever osteoarticular infection is chronic osteomyelitis. This is usually a result of contamination from a previous open fracture or prosthetic joints and vascular grafts. ${ }^{4}$ Adults usually present with an infected prosthetic joint and children with multifocal osteomyelitis. ${ }^{1}$

A review of this case revealed that the patient had previously travelled to Guangdong province in China and had contact with farm animals. This was the only potential source of infection from his history. A high index of suspicion is needed for diagnosis, as seen in this case, because a delay in treatment can lead to multiple surgeries. Despite being in an endemic area for spinal tuberculosis, ${ }^{5}$ other rarer causes of spondylodiscitis with granulomatous inflammation must be considered. These include other bacteria such as Brucellosis, melioidosis, actinomycosis, and Bartonella infections. Spirochetes, fungi, toxoplasmosis, and viruses such as infectious mononucleosis, cytomegalovirus, measles, and mumps are also potential causes. The presence of endovascular infection with spondylodiscitis with granulomatous inflammation and negative cultures should raise the alarm for potential $Q$ fever. Patients are 


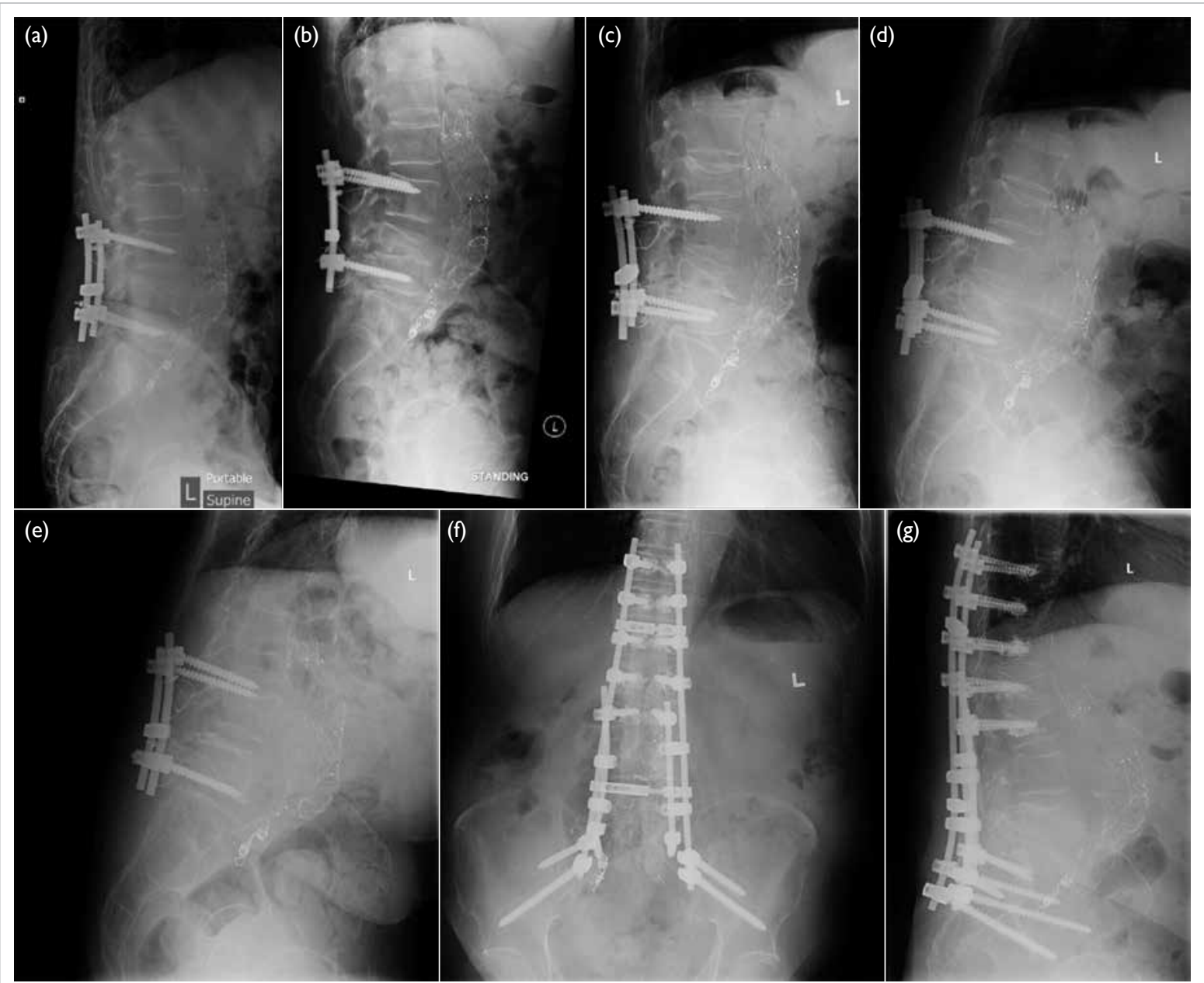

FIG 3. Same patient after L3-L5 laminectomy with posterior instrumented spinal fusion. Serial plain lateral radiographs (a) immediately after surgery, (b) I month after surgery showing progressive L4 collapse and gradual loosening of the screws, (c) 2 months after surgery, (d) 2.5 months after surgery showing kyphosis and screw cut-out, and (e) 3 months after surgery showing complete L 4 collapse and construct failure. Revision surgery was performed with stable construct at 2 years after surgery as seen on the (f) standing anteroposterior radiograph and $(\mathrm{g})$ standing lateral radiograph

commonly misdiagnosed and treated with prolonged antimicrobials and surgery without improvement.

\section{Author contributions}

Concept or design: JPY Cheung.

Acquisition of data: All authors

Analysis or interpretation of data: All authors.

Drafting of the manuscript: All authors.

Critical revision of the manuscript for important intellectual content: JPY Cheung.

All authors had full access to the data, contributed to the study, approved the final version for publication, and take responsibility for its accuracy and integrity.

\section{Conflicts of interest}

As an editor of the journal, JPY Cheung was not involved in the peer review process. Other authors have disclosed no conflicts of interest.

\section{Funding/support}

This case report received no specific grant from any funding agency in the public, commercial, or not-for-profit sectors.

\section{Ethics approval}

The patient was treated in accordance with the Declaration of Helsinki and provided informed consent for all treatments and procedures and consent for publication.

\section{References}

1. El-Mahallawy HS, Lu G, Kelly P, et al. Q fever in China: a systematic review, 1989-2013. Epidemiol Infect 2015;143:673-81.

2. Fantoni M, Trecarichi EM, Rossi B, et al. Epidemiological and clinical features of pyogenic spondylodiscitis. Eur Rev Med Pharmacol Sci 2012;16 Suppl 2:2-7.

3. Landais C, Fenollar F, Constantin A, et al. Q fever osteoarticular infection: four new cases and a review of the literature. Eur J Clin Microbiol Infect Dis 2007;26:341-7.

4. Melenotte C, Protopopescu C, Million M, et al. Clinical features and complications of Coxiella burnetii infections from the French National Reference Center for Q fever. JAMA Netw Open 2018;1:e181580.

5. Chan-Yeung M, Noertjojo K, Tan J, Chan SL, Tam CM. Tuberculosis in the elderly in Hong Kong. Int J Tuberc Lung Dis 2002;6:771-9. 